\title{
Derivación y validación de una ecuación para estimar el colesterol ligado a lipoproteínas de baja densidad en una población de Lima,
} Perú

\section{Derivation and validation of an equation to determine cholesterol-low density lipoproteins in a population of Lima, Peru}

\author{
Italo Moisés Saldaña Orejón ${ }^{1}$, Miguel Angel Benites Ricra², Justhin Andrés Chipana Huallpa² \\ ${ }^{1}$ Tecnólogo Médico-Químico farmacêutico, Hospital Nacional Edgardo Rebagliati Martins, Departamento de Patología Clínica, Servicio de Bioquímica. \\ ${ }^{2}$ Estudiante de pregrado de Tecnologia Médica, Universidad Alas Peruanas.
}

\section{Correspondencia}

Italo Moisés Saldaña Orejón imso_biochemical@yahoo.es Calle W 127 dpto. 102 Urb. Santa Rosa, Santiago de Surco.

Recibido, 1 abril 2016

Evaluado, 3 agosto 2016

Aceptado, 9 agosto 2016

Ningún conflicto de interes.

Citar como: Saldaña Orejón IM, Benites Ricra MA, Chipana Huallpa JA. Derivación y validación de una ecuación para estimar el colesterol ligado a lipoproteínas de baja densidad en una población de Lima, Perú. An Fac med. 2017;78(1):41-8. DOI: http://dx.doi.org/10.15381/ anales.v78i1.13020
An Fac med. 2017;78(1):41-8 / http://dx.doi.org/10.15381/anales.v7811.13020

\section{Resumen}

Introducción. Numerosos estudios han demostrado una fuerte asociación entre el aumento de la concentración sérica del colesterol de las lipoproteínas de baja densidad (LDLC) y la incidencia de la enfermedad coronaria (EC). Objetivos. Derivar y validar la performance de una nueva ecuación para estimar el LDLc. Diseño. Estudio observacional y descriptivo. Institución. Hospital Edgardo Rebagliati Martins, Lima, Perú. Material. Informes de perfil lipídico. Intervenciones. La muestra de 4644 informes de perfil lipídico extraídos de los archivos del laboratorio central, correspondiente a sujetos de ambos sexos, que presentaban edades entre 18 a 75 años, fue repartida en dos grupos. Del primer grupo conformado por 2136 informes se derivó la ecuación para estimar el LDLc, mediante análisis de regresión múltiple. El segundo grupo conformado por 2508 informes sirvieron para validar la performance de la nueva ecuación mediante la comparación con la medida del LDLc por el método directo homogéneo y estimado por la fórmula de Friedewald. Principal medida de resultados. Concentración sérica del LDLC. Resultados. La fórmula de Friedewald y la nueva ecuación infraestimaron el LDLc, obteniéndose sesgos de $14,01 \mathrm{mg} / \mathrm{dL}$ y $5,52 \mathrm{mg} / \mathrm{dL}$, respectivamente, con respecto al método directo. Los errores sistemáticos en los tres niveles de decisión clínica para el LDLc resultaron ser mayores para la fórmula de Friedewald $(12,6 \%, 12,0 \%$ y $11,7 \%)$ comparados a la nueva ecuación $(4,2 \%$, $5,7 \%$ y $6,7 \%)$. El factor de corrección de sesgo, que valora la exactitud, resultó ser más alto y más cerca de la línea de perfecta concordancia para la nueva ecuación, en toda la muestra y cuando esta se estratificó por niveles de triglicéridos. Conclusiones. La nueva ecuación ofrece una mayor exactitud y bajo error analítico comparada a la fórmula de Friedewald para estimar el LDLc, en los tres niveles de decisión clínica y en sueros con diferentes valores de triglicéridos.

Palabras clave. Lipoproteinas de Baja Densidad; Enfermedad Coronaria; Dislipidemias.

Abstract

Introduction: Various studies have shown a strong association between the increase in cholesterol low density lipoproteins (LDLC) serum concentration and the incidence of coronary heart disease. Objectives: To derive and validate the performance of a new equation to estimate the LDLC. Design: Observational and descriptive study. Institution: Hospital Edgardo Rebagliati Martins, Lima, Peru. Material: 4644 lipid profile reports taken from the files of the hospital's central laboratory, belonging to subjects of bot. sexes, between 18 and 75 years old. Interventions: The sample consisted of 4644 lipid profile reports obtained from the files of the hospital's central laboratory, and belonged to subjects of both sexes aged 18-75 years. It was divided into two groups: the first group was represented by 2136 reports, and was used to derive the equation to estimate the LDLC by using multiple regression analysis; the second group comprised 2508 reports that served to validate the performance of the new equation compared with the LDLc measurement by the homogeneous direct method and estimated by the Friedewald formula. Main outcome measure: LDLc serum concentration. Results: The Friedewald formula and the new equation underestimated the LDLc with a shortfall of $14.01 \mathrm{mg} / \mathrm{dL}$ and 5.52 $\mathrm{mg} / \mathrm{dL}$, respectively, with regard to the direct method. Systematic errors in the three clinical decision levels for $L D L$ cholesterol were found to be higher for the Friedewald formula $(12.6 \%, 12.0 \%$, and $11.7 \%)$ compared to the new equation $(4.2 \%, 5.7 \%$ and $6.7 \%)$. The bias correction factor, which assessed accuracy, turned out to be higher and closer to the line of perfect match for the new equation, both in the whole sample and when the entire sample was stratified by levels of triglycerides. Conclusions: The new equation provided greater accuracy and lower analytical error compared to the Friedewald formula to estimate the LDL cholesterol in the three clinical decision levels and with different values of serum triglycerides.

Keywords: Low Density Lipoproteins; Coronary Heart Disease; Dyslipidemias. 


\section{INTRODUCCIÓN}

Los lípidos, por su carácter hidrofóbico, no se encuentran circulando libres en el plasma, sino que se unen a proteínas, conformando complejos macromoleculares solubles denominados lipoproteínas. Se reconocen 4 tipos mayores de lipoproteínas clasificadas según su tamaño, composición y densidad. Estas son los quilomicrones y las lipoproteínas de muy baja densidad, de baja densidad y de alta densidad ${ }^{(1)}$.

Diversos estudios epidemiológicos y clínicos han demostrado de manera consistente que el incremento de la concentración en sangre del colesterol ligado a las lipoproteínas de baja densidad (LDLC) es un factor de riesgo importante en el desarrollo de la enfermedad cardíaca coronaria. Por lo tanto, su estimación exacta y precisa es de suma importancia para el diagnóstico, tratamiento y seguimiento de los pacientes con dislipidemias ${ }^{(2,3)}$.

Dada la importancia clínica del LDLC, los laboratorios clínicos deberían disponer de métodos sencillos, fiables y estandarizados para su medida rutinaria. El método de beta-cuantificación considerado como de referencia precisa de una ultracentrifugación, además de ser una metodología muy laboriosa que consume mucho tiempo y utiliza grandes volúmenes de muestra.

En los laboratorios clínicos, la cuantificación del LDLc hasta hace unos años se realizaba casi exclusivamente por métodos de precipitación, usando agentes químicos como heparina, polivinilsulfato, sulfato de dextrán o polímeros anfipáticos inespecíficos. Estos ensayos, además de laboriosos y tediosos, presentan ciertas limitaciones, pues existen evidencias que muestran que en casos de hipertrigliceridemia se corre el riesgo de precipitar otras fracciones ricas en colesterol presentes en el sobrenadante, todo ello, sumado a que estos ensayos no pueden automatizarse completamente, representando desventajas apreciables en cuanto a precisión, exactitud o especificidad $^{(4,5)}$.

La necesidad de mejorar la eficacia diagnóstica de los métodos de precipitación, tuvo como consecuencia el desa- rrollo de métodos denominados homogéneos directos, que emplean reactivos específicos para medir selectivamente el LDLc en las muestras de sangre, el cual parece aportar notables ventajas con respecto al método de precipitación. Pero la accesibilidad a estos métodos todavía es limitada en los laboratorios clínicos, debido los altos costos de los reactivos, por lo que no son utilizados de forma rutinaria.

Por ello, la mayoría de laboratorios de nuestro país estima indirectamente la concentración del LDLc mediante la fórmula de Friedewald, que precisa de la concentración del colesterol total (CT), de las lipoproteínas de alta densidad (HDLC) y de los triglicéridos (TG). Aunque es un método de bajo costo y fácil de realizar, esta fórmula no debe ser aplicada cuando las concentraciones de TG sean superiores a $400 \mathrm{mg} / \mathrm{dL}$ o en pacientes con disbetalipoproteinemia. Además, diversos estudios demuestran la inexactitud de esta estimación en ciertas patologías como diabetes, hepatopatías e insuficiencia renal ${ }^{(6,7)}$.

A pesar de las desventajas técnicas de la fórmula de Friedewald, es difícil desplazarlo de la práctica clínica a menos que se desarrolle un método con claras ventajas en el rendimiento diagnóstico.

El objetivo del presente estudio fue derivar una ecuación para estimar el LDLc por análisis de regresión lineal mutivariante y validar la performance de dicha ecuación mediante la comparación con el método directo y la fórmula de Friedewald en diferentes niveles de triglicéridos, utilizando los datos de los perfiles de lípidos de un centro hospitalario de Lima, Perú.

\section{MÉTODOS}

El presente es un estudio observacional y descriptivo, cuyo material estuvo conformado por 4644 informes de perfil lipídico extraídos de los archivos del laboratorio central del Hospital Edgardo Rebagliati Martins, de EsSalud, Lima, Perú, correspondientes a sujetos de ambos sexos, que presentaban edades entre 18 a 75 años. Fueron repartidos en dos grupos: a) el primer grupo estuvo conformado por 2136 informes provenientes de sujetos a los cuales se les realizó la medición de colesterol total, triglicéridos y las lipoproteínas HDLc y LDLc mediante métodos homogéneos directos, durante el período de enero a marzo de 2015; sus resultados derivaron en la ecuación para estimar el LDLC, basado en el análisis de regresión lineal mutivariante, considerando al LDLc como variable dependiente y al colesterol total, triglicéridos y HDLc como variables independientes; b) el segundo grupo lo conformaron 2508 resultados provenientes de sujetos que se sometieron a la medición del perfil lipídico, durante el período de abril a julio de 2015, cuyos resultados sirvieron para validar la performance de la ecuación propuesta, mediante la comparación con el método directo y la fórmula de Friedewald ${ }^{(8,9)}$.

Los informes que no presentaban todos los resultados completos del perfil lipídico o que tenían alguna observación con respecto a la calidad de la muestra, fueron excluidos de la investigación.

La recolección de las muestras se realizó en tubos al vacío con gel separador. Luego de la retracción del coágulo, se centrifugó 10 minutos a 3500 RPM, para obtener el suero. Después de la centrifugación, el suero se utilizó para el análisis del perfil de lípidos.

Se determinó el efecto del incremento de los triglicéridos sobre las concentraciones de LDLc estimadas por la fórmula de Friedewald y la nueva ecuación. Para ello, se estratificaron las 2508 muestras en 5 subgrupos en función de sus concentraciones de triglicéridos: $\leq 100 \mathrm{mg} / \mathrm{dL}$ (1,13 mmol/L) ( $\mathrm{n}=690) ; 101-200 \mathrm{mg} / \mathrm{dL}$ (1,14-2,26 mmol/L) ( $\mathrm{n=1} 2 \mathrm{299}) ; 201-300$ $\mathrm{mg} / \mathrm{dL}(2,27-3,39 \mathrm{mmol} / \mathrm{L})(\mathrm{n}=366) ; 301$ $400 \mathrm{mg} / \mathrm{dL}(3,40-4,52 \mathrm{mmol} / \mathrm{L})(\mathrm{n}=94)$; $\geq 401 \mathrm{mg} / \mathrm{dL}(4,53 \mathrm{mmol} / \mathrm{L})(\mathrm{n}=59)$.

Según el National Cholesterol Education Program (NCEP), el requisito del mínimo de calidad analítica para la determinación del LDLc, no debe sobrepasar el $\pm 12 \%$ de error total ${ }^{(6,27)}$. Para la evaluación de este último requisito determinamos el número y porcentaje de resultados estimados por la fórmula de Friedewald y la nueva ecuación con una diferencia porcentual menor o igual al 
$12 \%$ con respecto al método de determinación directa.

Los perfiles de lípidos fueron valorados en el autoanalizador ADVIA1800 con reactivos y calibradores de SIEMENS ${ }^{\circledR}$. Los métodos analíticos se realizaron según las indicaciones del fabricante. Los niveles séricos de colesterol total fueron medidos mediante una técnica enzimática basada en la conversión mediada por colesterol-esterasa y colesterol-oxidasa, seguida de un punto final de Trinder $(10,11)$. La concentración sérica de triglicéridos se determinó mediante la técnica enzimática en tres pasos de Fossati, con un punto final de Trinder ${ }^{(8,9)}$. Los niveles de HDLc y LDLc fueron medidos por el método automatizado directo basado en que un detergente específico solubiliza las lipoproteínas que no son de interés, las cuales son hidrolizadas por la colesterol esterasa y la colesterol oxidasa mediante una reacción no formadora de color. El segundo detergente, presente en el reactivo $B$, solubiliza las lipoproteínas de interés y se cuantifica espectrofotométricamente mediante las reacciones acopladas descritas para el colesterol total ${ }^{(12,13)}$.

El analizador fue calibrado previamente al estudio, de acuerdo a las recomendaciones del fabricante. El programa de control de calidad interno incluyó la evaluación de sueros controles BIO-RAD ${ }^{\circledR}$ de dos niveles de decisión, pertenecientes a los lotes 14491 y 14492 respectivamente, que se procesaron diariamente y una muestra mensual de un control de calidad externo internacional (RIQAS), con lote de referencia RQ9128 ciclo 12B. El método directo para la determinación del LDLc, considerado en este estudio como el de referencia, presentó la imprecisión analítica intraserial de 2,54\% y $1,31 \%$ para los dos niveles de los sueros controles.

Para el análisis estadístico se aplicó la prueba de Kolmogorov-Smirnov, para corroborar la normalidad de la muestra.

Para la descripción de la variable sexo, se calculó los porcentajes, mientras que las variables edad y parámetros lipídicos fueron expresados como rango y media \pm desviación estándar (DE). Las correlaciones para asociaciones entre variables fueron realizadas según el coeficiente rho de Spearman.

Para establecer la forma en la que los valores de la ecuación a evaluar varían en función de los valores del método considerado de referencia, se empleó la prueba de regresión de Passing-Bablock, expresada por la ecuación $y=b x+a$, donde ' $b$ ' es la pendiente de la recta de regresión que representa el error proporcional, y 'a' el valor de la ordenada en el origen (corte de la recta en el eje de ordenadas) que representa el error constante.

Las diferencias entre medias de los valores de LDLc obtenidas por el método directo y estimado por las fórmulas, fueron analizadas mediante la prueba de rangos de Wilcoxon para muestras relacionadas.

El análisis de concordancia entre dos pruebas se realizó con el método de Bland-Altman, que consiste en representar gráficamente las diferencias entre cada pareja de valores de LDLc estimado y medido, frente a la media de los mismos. El 95\% de las diferencias cae entre dos límites que definen el intervalo de concordancia: el límite inferior, el cual es el promedio de la diferencia menos dos desviaciones estándar, y el límite superior, el cual es el promedio de la diferencia más dos desviaciones estándar ${ }^{(14)}$.

Por último, para reforzar el estudio de la concordancia recurrimos al estadístico coeficiente de correlación concordancia de Lin (CCC). Este coeficiente combina dos elementos, el coeficiente de correlación ( $r$ ) que evalúa la precisión y el factor de corrección de sesgo ( $\mathrm{Cb}$ ), que valora la exactitud, es decir, qué tan lejos se desvían los datos observados por dos métodos con respecto a la línea a partir del origen y a $45^{\circ}$ en un plano cartesiano, que corresponde a la línea de perfecta concordancia. Dicho coeficiente califica la fuerza de acuerdo como casi perfecta para valores mayores a 0,99, sustancial de 0,95 a 0,99, moderado de 0,90 a 0,95, y pobre cuando está por debajo de 0,90. La realización de las pruebas estadísticas antes mencionadas fue valorada mediante el software MedCalc versión 15.11.4 y el software SPSS versión 22, considerándose significativos valores de $p<0,05$.

\section{RESULTADOS}

Los resultados de LDLC obtenidos por el método directo y las ecuaciones de estimación, no presentaron una distribución normal $(p<0,05)$.

En la tabla 1 se expone los resultados descriptivos de la edad, el sexo y los parámetros lipídicos de los dos grupos que conformaron el estudio.

De los datos del perfil de lípidos del primer grupo se derivó la ecuación para estimar el LDLc, basado en el análisis de regresión lineal múltiple. En la tabla 2 se muestra el valor de la constante y los respectivos coeficientes de las variables, además de su grado de significación $p<$ 0,0001 . De esto último se puede afirmar que existe un efecto significativo lineal entre el LDLc y las variables colesterol total, triglicéridos y HDLc, lo que nos permitió desarrollar la nueva ecuación para estimar el LDLc en $\mathrm{mg} / \mathrm{dL}$ :

$$
\begin{aligned}
\mathrm{LDLC}= & 0,974 \times \mathrm{CT}-0,160 \times \mathrm{TG}- \\
& 0,968 \times \mathrm{HDLC}+5,361
\end{aligned}
$$

Una vez desarrollada la ecuación, se procedió a su validación mediante estudio de concordancia con el método directo, considerado el de referencia, y la fórmula de Friedewald en $\mathrm{mg} / \mathrm{dL}$ :

$$
\mathrm{LDLC}=\mathrm{CT}-\mathrm{TG} / 5-\mathrm{HDLC}
$$

Respecto a la linealidad y dispersión constante, los dos métodos de estimación seguían una relación lineal con el ensayo directo, obteniéndose un coeficiente de correlación de 0,947 ( $p<0,0001)$ para la fórmula de Friedewald y 0,954 ( $p$ $<0,0001)$ para la nueva ecuación.

La regresión de Passing y Bablok muestra la siguiente ecuación de regresión lineal para la fórmula de Friedewald, tal que: LDLc Friedewald (y) =0,90 LDLC directo $-2,64$. Mientras que para el caso de la nueva fórmula, la ecuación de regresión lineal resultó: LDLc Nueva ecuación = 0,89 LDLc directo + 6,84.

De las ecuaciones obtenidas se percibe que ninguna de las fórmulas para estimar el LDLc tiene una identidad perfecta $(y=x)$ con la medición directa del LDLc. Sin embargo, la desviación estándar residual $\pm 1,96 \mathrm{DE}$ de las rectas de regresión

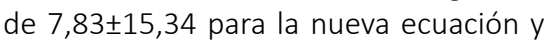


Tabla 1. Características y parámetros lipídicos valorados en el autoanalizador ADVIA1800 por métodos directos de los grupos de derivación y validación de la nueva ecuación.

\begin{tabular}{|c|c|c|c|c|}
\hline \multirow{3}{*}{$\begin{array}{l}\text { Sexo } \\
\text { masculino/femenino (\%) }\end{array}$} & \multicolumn{2}{|c|}{ Grupo de derivación de la ecuación (n=2136) } & \multicolumn{2}{|c|}{ Grupo de validación de la ecuación $(n=2508)$} \\
\hline & \multicolumn{2}{|c|}{$868 / 1268(41 / 59)$} & \multicolumn{2}{|c|}{$1022 / 1486(41 / 59)$} \\
\hline & Media $\pm D E$ & Rango & Media $\pm D E$ & Rango \\
\hline Edad (años) & $58,9 \pm 16,2$ & 18 a 75 & $60,7 \pm 16,2$ & 18 a 75 \\
\hline Colesterol total (mmol/L) & $4,80 \pm 1,25$ & 1,73 a 12,10 & $4,48 \pm 1,70$ & 1,60 a 10,99 \\
\hline Colesterol total (mg/dL) & $185,65 \pm 48,48$ & 67 a 468 & $173,42 \pm 65,70$ & 62 a 425 \\
\hline Triglicéridos (mmol/L) & $1,90 \pm 1,22$ & 0,47 a 11,81 & $1,74 \pm 1,01$ & 0,34 a 9,59 \\
\hline Triglicéridos (mg/dL) & $168,60 \pm 107,66$ & 42 a 1046 & $154,53 \pm 89,85$ & 30 a 849 \\
\hline $\mathrm{HDLc}(\mathrm{mmol} / \mathrm{L})$ & $1,14 \pm 0,33$ & 0,44 a 2,33 & $1,17 \pm 0,34$ & 0,44 a 2,33 \\
\hline $\mathrm{HDLc}(\mathrm{mg} / \mathrm{dL})$ & $44,01 \pm 12,61$ & 17 a 90 & $45,34 \pm 13,31$ & 17 a 90 \\
\hline LDLc medición directa (mmol/L) & $3,02 \pm 1,17$ & 0,62 a 8,12 & $2,87 \pm 1,21$ & 0,62 a 6,85 \\
\hline LDLc medición directa (mg/dL) & $116,77 \pm 45,20$ & 24 а 314 & $110,90 \pm 46,74$ & 24 a 265 \\
\hline
\end{tabular}

DE: desviación estándar, HDLc: colesterol ligado a lipoproteína de alta densidad, LDLc: colesterol ligado a lipoproteína de baja densidad.

$8,24 \pm 16,14$ para la fórmula Friedewald, muestran que la desviación de la línea $y=x$ fue menor para la nueva ecuación (figura 1).

La ecuación de regresión lineal permite obtener el error sistemático en los puntos de interés médico, de tal forma que sustituyendo $X$ por los valores de concentración críticos se obtiene los errores predichos, en los tres niveles de decisión clínica para el LDLc $(100$ mg/dL,
$130 \mathrm{mg} / \mathrm{dL}$ y $160 \mathrm{mg} / \mathrm{dL})^{(6)}$. Los errores sistemáticos calculados en los tres niveles de decisión clínica para la nueva ecuación, -4,16 mg/dL (4,2\%); -7,46 mg/ $\mathrm{dL}(5,7 \%)$ y $-10,76 \mathrm{mg} / \mathrm{dL}(6,7 \%)$, frente a la fórmula de Friedewald, $-12,64 \mathrm{mg} /$ $\mathrm{dL}(12,6 \%),-15,64 \mathrm{mg} / \mathrm{dL}(12 \%)$ y-18,64 $\mathrm{mg} / \mathrm{dL}(11,7 \%)$, evidencian que la nueva ecuación posee una mayor veracidad para estimar el LDLc en los puntos de interés clínico.

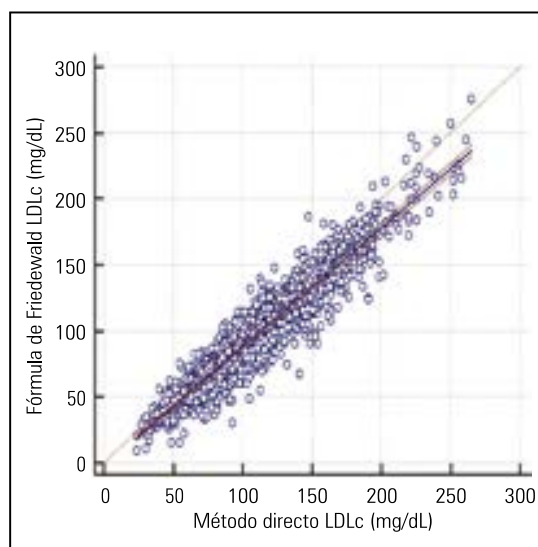

Método directo vs Fórmula de Friedewald Ecuación de la línea de regresión: $y=0,90 x-2,64$ Intercepto: -2,64, IC 95\% $-3,89$ a $-1,50$ Pendiente: 0,90, IC 95\% 0,89 a 0,91

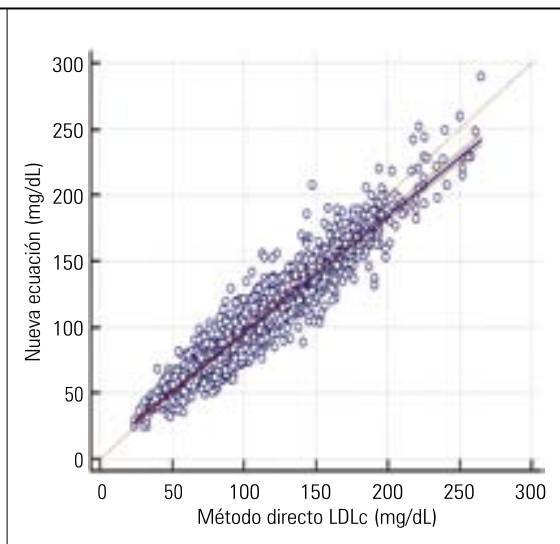

Método directo vs Nueva ecuación Ecuación de la línea de regresión: $y=0,89 x+6,84$ Intercepto: 6,84, IC 95\% 5,67 a 7,90 Pendiente: 0,89 , IC $95 \%$ 0,88 a 0,90

Figura 1. Recta de regresión lineal entre la determinación directa y las estimaciones del LDLc por la ecuación de Friedewald y la nueva ecuación.

Las medias de los niveles de LDLc obtenidos mediante el método directo, la fórmula de Friedewald y la nueva ecuación en el total de la muestra y agrupados en diferentes niveles de triglicéridos, se muestran en la tabla 3.

En el total de la muestra, la media de los valores de LDLc medido por el método directo y estimado por la fórmula de Friedewald y la nueva ecuación fue 111,17 $\mathrm{mg} / \mathrm{dL}, 97,16 \mathrm{mg} / \mathrm{dL}$ y 105,65 mg/dL, respectivamente. Hay que indicar que la media de las determinaciones por la nueva ecuación produjo resultados más cercanos a la media de los resultados determinados por el método directo en toda la muestra y en los subgrupos clasificados por el nivel de triglicéridos, a comparación de la fórmula de Friedewald, cuya media de estimaciones resultó más alejada del método directo (figura 2).

Se obtuvieron diferencias significativas en el análisis de comparación de medias entre el método directo y las ecuaciones de estimación del LDLc en total del grupo y cuando fueron agrupadas en diferentes niveles de triglicéridos ( $p<0,0001)$, a excepción de los grupos pertenecientes a la nueva ecuación, con valores de triglicéridos entre 301 a $400 \mathrm{mg} / \mathrm{dL}(p=0,052)$ y mayor a $401 \mathrm{mg} / \mathrm{dL}(\mathrm{p}=0,357)$.

La diferencia promedio entre el método de medición directa y las ecuaciones de estimación correspondió a -14,01 
Tabla 2. Coeficientes obtenidos por regresión lineal múltiple de las variables descritas con relación al LDLc determinado por el método directo homogéneo.

\begin{tabular}{cccc} 
Variables & Coeficiente & Error estándar & $\mathrm{p}^{*}$ \\
& $\mathrm{mg} / \mathrm{dL}$ & & - \\
Constante & 5,361 & - & $<0,0001$ \\
Colesterol total $(\mathrm{mg} / \mathrm{dL})$ & 0,974 & 0,007 & $<0,0001$ \\
Triglicéridos $(\mathrm{mg} / \mathrm{dL})$ & $-0,160$ & 0,003 & $<0,0001$ \\
HDLc $(\mathrm{mg} / \mathrm{dL})$ & $-0,968$ & 0,027 & - \\
& $\mathrm{mmol} / \mathrm{L}$ & & $<0,0001$ \\
\hline Constante & 0,139 & - & $<0,0001$ \\
Colesterol total $(\mathrm{mmol} / \mathrm{L})$ & 0,974 & 0,007 & $<0,0001$ \\
\hline Triglicéridos $(\mathrm{mmol} / \mathrm{L})$ & $-0,367$ & 0,007 & 0,027 \\
HDLc $(\mathrm{mmol} / \mathrm{L})$ & $-0,968$ & 0
\end{tabular}

LDLc: Colesterol ligado a lipoproteína de baja densidad, HDLc: colesterol ligado a lipoproteína de alta densidad. * $p$ corresponde al grado de significación. $\mathrm{mg} / \mathrm{dL}$ para la fórmula de Friedewald, mientras que para la nueva ecuación resultó $-5,52 \mathrm{mg} / \mathrm{dL}$. Tanto la ecuación de Friedewald como la nueva fórmula infraestimaron el LDLC comparadas con el método directo. Sin embargo, la nueva fórmula presentó un menor sesgo absoluto en toda la muestra y en todos los grupos clasificados de acuerdo a los niveles de triglicéridos.

La determinación del número y porcentaje de estimaciones de LDLc con una diferencia porcentual menor o igual al $12 \%$ con referencia al método de determinación directa, reveló que 1868 estimaciones correspondiente al $74,5 \%$ caen dentro de $\pm 12 \%$ para la nueva ecua-

Tabla 3. Media de los valores de LDLc, sesgo absoluto y porcentual, coeficiente de correlación concordancia (CCC), factor de corrección de sesgo (Cb) y coeficiente de correlación ( $r$ ), obtenido por las fórmulas de estimación en comparación con el método directo en toda la muestra y estratificado en diferentes niveles de triglicéridos.

\begin{tabular}{|c|c|c|c|c|c|c|}
\hline & $\begin{array}{l}\text { Media } \pm \text { DS } \\
\quad(\mathrm{mg} / \mathrm{dL})\end{array}$ & $\begin{array}{c}\text { Sesgo* } \pm \text { DS } \\
(\mathrm{mg} / \mathrm{dL})\end{array}$ & Sesgo $(\%)^{* *}$ & $\begin{array}{c}\text { CCC } \\
(\text { IC 95\%) }\end{array}$ & $\begin{array}{c}\mathrm{Cb} \\
\text { (exactitud) }\end{array}$ & $\begin{array}{c}\mathrm{r} \\
\text { (precisión) }\end{array}$ \\
\hline \multicolumn{7}{|c|}{ Todas las muestras ( $n=2508$ ) } \\
\hline Método directo & $111,17 \pm 38,06$ & - & - & - & - & - \\
\hline Fórmula de Friedewald & $97,16 \pm 35,22$ & $-14,01 \pm 11,97$ & 12,60 & $0,882(0,875-0,890)$ & 0,93 & 0,95 \\
\hline Nueva fórmula & $105,65 \pm 34,75$ & $-5,52 \pm 11,54$ & 4,97 & $0,939(0,935-0,943)$ & 0,98 & 0,95 \\
\hline \multicolumn{7}{|l|}{$\mathrm{TG} \leq 100 \mathrm{mg} / \mathrm{dL}(\mathrm{n}=690)$} \\
\hline Método directo & $96,45 \pm 31,53$ & - & - & - & - & - \\
\hline Fórmula de Friedewald & $86,78 \pm 28,76$ & $-9,67 \pm 8,55$ & 10,03 & $0,913(0,902-0,923)$ & 0,95 & 0,96 \\
\hline Nueva fórmula & $92,91 \pm 28,12$ & $-3,54 \pm 8,58$ & 3,67 & $0,952(0,945-0,958)$ & 0,99 & 0,97 \\
\hline \multicolumn{7}{|c|}{ TG de 101 a $200 \mathrm{mg} / \mathrm{dL}(\mathrm{n}=1299)$} \\
\hline Método directo & $114,61 \pm 37,63$ & - & - & - & - & - \\
\hline Fórmula de Friedewald & $100,44 \pm 34,87$ & $-14,17 \pm 10,72$ & 12,36 & $0,889(0,879-0.898)$ & 0,93 & 0,96 \\
\hline Nueva fórmula & $108,41 \pm 34,05$ & $-6,19 \pm 10,74$ & 5,40 & $0,941(0,907-0,934)$ & 0,98 & 0,96 \\
\hline \multicolumn{7}{|c|}{ TG de 201 a $300 \mathrm{mg} / \mathrm{dL}(\mathrm{n}=366)$} \\
\hline Método directo & $124,39 \pm 40,03$ & - & - & - & - & - \\
\hline Fórmula de Friedewald & $105,68 \pm 37,91$ & $-18,71 \pm 13,84$ & 15,04 & $0,840(0,813-0,863)$ & 0.90 & 0,94 \\
\hline Nueva fórmula & $116,85 \pm 37,03$ & $-7,54 \pm 13,78$ & 6,06 & $0,919(0,902-0,933)$ & 0,98 & 0,94 \\
\hline \multicolumn{7}{|c|}{ TG de 301 a $400 \mathrm{mg} / \mathrm{dL}(\mathrm{n}=94)$} \\
\hline Método directo & $119,79 \pm 46,45$ & - & - & - & - & - \\
\hline Fórmula de Friedewald & $100,06 \pm 46,37$ & $-19,72 \pm 16,68$ & 16,46 & $0,857(0,803-0,898)$ & 0,92 & 0,94 \\
\hline Nueva fórmula & $114,94 \pm 45,07^{\mathrm{a}}$ & $-4,85 \pm 16,53$ & 4,05 & $0,930(0,896-0,952)$ & 0,99 & 0,94 \\
\hline \multicolumn{7}{|l|}{$\mathrm{TG} \geq 401 \mathrm{mg} / \mathrm{dL}(\mathrm{n}=59)$} \\
\hline Método directo & $112,07 \pm 38,84$ & - & - & - & - & - \\
\hline Fórmula de Friedewald & $88,94 \pm 45,24$ & $-23,13 \pm 24,46$ & 20,64 & $0,721(0,600-0,810)$ & 0,86 & 0,84 \\
\hline Nueva fórmula & $109,51 \pm 43,63^{a}$ & $-2,55 \pm 23,98$ & 2,28 & $0,830(0,733-0,984)$ & 0,99 & 0,84 \\
\hline
\end{tabular}

LDLC: colesterol ligado a lipoproteínas de baja densidad, TG: triglicéridos.

IC 95\%: intervalo de confianza al 95\%

*Media de las diferencias entre el LDLc estimado por las fórmulas y determinado por el método directo.

** (Sesgo/LDLc método directo) $\times 100$

a: No se observa diferencia significativa entre el método directo y la fórmula de estimación del LDLc. 


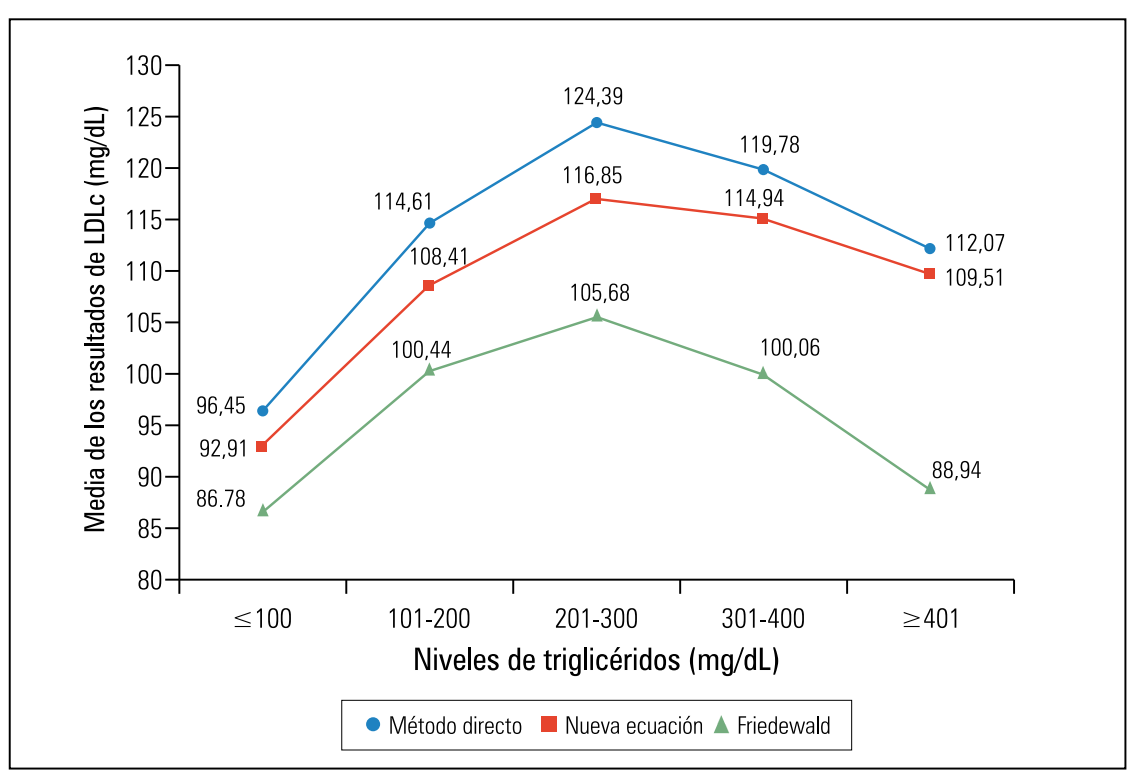

Figura 2. Comparación entre los valores medios de LDLc obtenidos por el método directo y las fórmulas de estimación de Friedewald y la nueva ecuación, en diferentes niveles de triglicéridos.

ción, mientras que solo 1183 resultados $(47,2 \%)$ estimados por la fórmula de Friedewald presentaron una diferencia porcentual menor o igual al $12 \%$.

La estimación del intervalo de concordancia del 95\% de la diferencia promedio por el análisis de Bland - Altman resulto entre $-9,4$ y $37,5 \mathrm{mg} / \mathrm{dL}$ para la fórmula de Friedewald y de $-17,1$ a $28,1 \mathrm{mg} / \mathrm{dL}$ para la nueva ecuación. Ambos intervalos de confianza incluían el valor cero; sin embargo, la amplitud de los límites de acuerdo fue más estrecha para la nueva ecuación (figura 3).

El coeficiente de concordancia calculada mediante el CCC de Lin entre la determinación directa y la estimación del LDLC mediante la fórmula de Friedewald fue 0,882 (IC95\% 0,875 a 0,890), correspondiente un grado de concordancia pobre. Los componentes del coeficiente fueron, precisión 0,950 y factor de corrección de sesgo (exactitud) de 0,929, mientras que para el caso de la concordancia entre la determinación directa y la nueva ecuación, el CCC de Lin fue 0,939 (IC95\% 0,943 a 0,951), correspondiente un grado de concordancia moderada. A su vez, los componentes del coeficiente fueron, precisión 0,953 y el factor de corrección de sesgo (exactitud) 0,985. La exactitud lúa la precisión, para ambas fórmulas de estimación de LDLc, en los 5 subgrupos estratificados, en función a los niveles de triglicéridos.

\section{DISCUSIÓN}

Las principales guías de prácticas clínicas consideran que los niveles de LDLc son un criterio importante para determinar el riesgo de adquirir enfermedad cardiaca coronaria, establecer el ajuste de la dieta, la intensidad del tratamiento farmacológico y la monitorización de las dislipidemias. Por lo tanto, su estimación exacta es de suma importancia ${ }^{(15,16)}$.

La mayoría de los laboratorios clínicos estiman las concentraciones de LDL usando la fórmula de Friedewald. Pero esta fórmula presenta ciertas limitaciones. Con el fin de mejorar la exactitud de dicha estimación, se ha propuesto diversas modificaciones de la fórmula original, pero ninguna ha aportado pruebas suficientes para reemplazarla ${ }^{(17,18)}$.

Las diferentes fórmulas propuestas en diversos estudios para estimar el LDLC han sido validadas para una población particular. En este estudio hemos derivado y validado la performance de una nueva ecuación basada en los perfiles de el coeficiente de correlación (r) que eva-

En la tabla 3 se muestra los valores de CCC de Lin, el factor de corrección de sesgo ( $\mathrm{Cb}$ ) que valora la exactitud y de la nueva ecuación $(\mathrm{Cb})$ resultó ser más alta y más cerca de la línea de perfecta concordancia $(\mathrm{Cb}=1)$, en comparación con la fórmula de Friedewald.

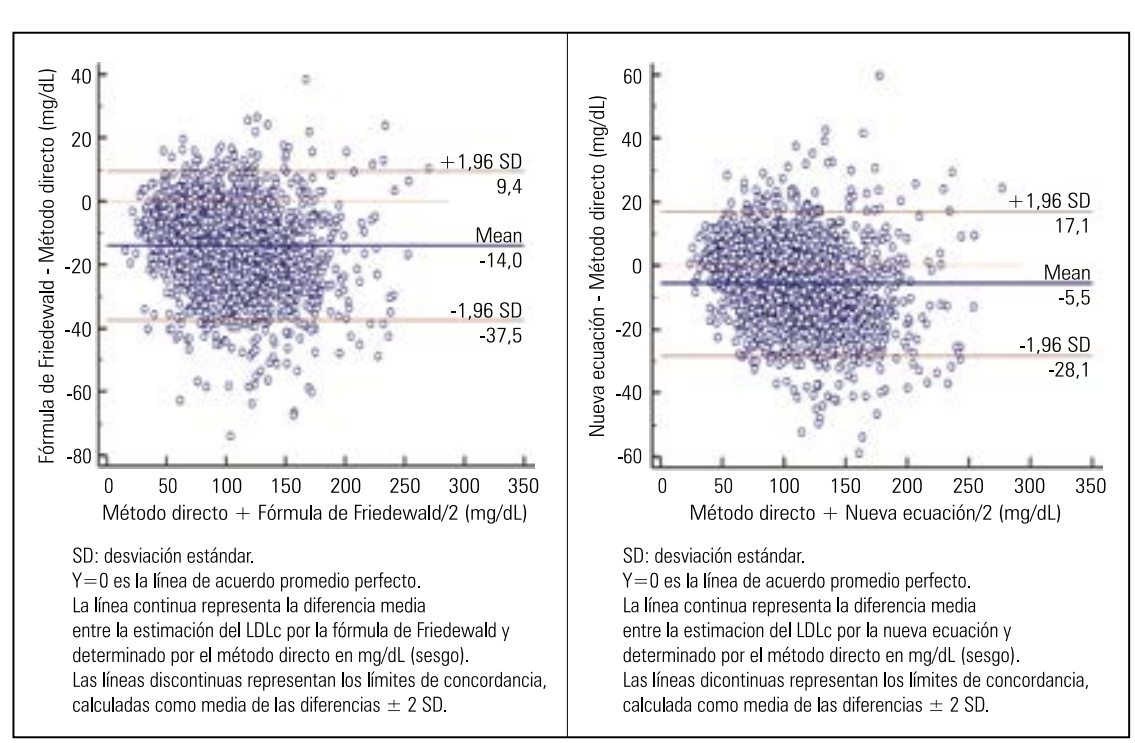

Figura 3. Gráfico de Bland-Altman entre la determinación directa y las estimaciones del LDLc por la ecuación de Friedewald y la nueva ecuación. 
lípidos provenientes de un centro hospitalario de Lima (19-21).

En el total de sujetos del estudio, la fórmula de Friedewald y la nueva ecuación infraestimaron el LDLc, obteniéndose sesgos absolutos de 14,01 mg/dL y $5,52 \mathrm{mg} / \mathrm{dL}$, respectivamente, resultados que coinciden con otros estudios que también utilizaron el análisis de regresión múltiple para derivar su ecuación, pero validado en otra población ${ }^{(22,23)}$

Como esperábamos, encontramos aumento de los sesgos absolutos y porcentuales para estimar el LDLc mediante la fórmula de Friedewald, por efecto del incremento de los niveles de TG, resultados que coinciden con otros estudios, lo que confirma la relación entre el error estimado y la concentración de los TG para la fórmula de Friedewald (24-26). Mientras que para el caso específico de la nueva ecuación, estos sesgos fueron menores y más uniformes, indicándonos que la influencia de la concentración de los triglicéridos sobre la estimación del LDLc por la nueva ecuación es mínima.

Según el National Cholesterol Education Program (NCEP), el requisito mínimo de calidad analítica para la determinación del LDLc no debe sobrepasar el $\pm 12 \%$ de error total ${ }^{(6,27)}$. En nuestro estudio, de las 2508 determinaciones de LDLc, 52,8\% y $25,5 \%$ de las estimaciones por la fórmula de Friedewald y la nueva ecuación, respectivamente, presentaron una diferencia porcentual mayor a $\pm 12 \%$ con respecto al método directo, lo que evidencia un menor error de estimación de la nueva ecuación a comparación de la fórmula de Friedewald para estimar el LDLc, resultados que concuerdan con los estudios realizados por Saiedullah y Chowdhury en una población de Bangladesh ${ }^{(23,28)}$.

Por lo general, se acepta que la fórmula de Friedewald no es confiable cuando los niveles de TG exceden los 400 mg/ dL. Sin embargo, los sesgos porcentuales hallados mayores a $15 \%$ evidencian la inexactitud de dicha fórmula para concentraciones de TG mayores a 200 mg/ $\mathrm{dL}$ (tabla 3).

Las especificaciones de calidad deben ser definidas a concentraciones de nivel de decisión clínica, que son los niveles de concentración del analito donde existe la mayor probabilidad de cometer algún error diagnóstico. Nuestros datos indican que los errores sistemáticos determinados por la prueba de regresión lineal en los tres niveles de decisión clínica para el LDLc, en el caso de la fórmula de Friedewald resultaron mayores al 10\%, a comparación de la nueva ecuación cuyos errores sistemáticos oscilaron entre 4,2\% y $6,7 \%$, evidenciando que la nueva ecuación posee una mayor veracidad para estimar el LDLc en los puntos de interés clínico ${ }^{(6)}$

Al aplicar el análisis de coeficiente de correlación concordancia de Lin (CCC), se obtuvieron valores de dicho coeficiente por debajo de 0,90, correspondiente a un grado de acuerdo pobre, en el total de la muestra y cuando se estratifico por niveles de triglicéridos para el caso de la fórmula de Friedewald. Es de notar que en el grupo de mayor nivel de triglicéridos ( $\geq 400 \mathrm{mg} / \mathrm{dL}$ ), este coeficiente disminuyó drásticamente a un valor de 0,72, lo que confirma el efecto de la concentración de triglicéridos sobre el error en la estimación del LDLc para dicha fórmula.

El estadístico coeficiente de correlación concordancia de Lin (CCC), combina dos elementos, el coeficiente de correlación (r) que evalúa la precisión y el factor de corrección de sesgo (Cb), que valora la exactitud. Para el caso de nuestro estudio encontramos una precisión muy similar para ambas ecuaciones, mientras que la exactitud de la nueva ecuación resultó ser más alta y más cerca de la línea de perfecta concordancia $(\mathrm{Cb}=1)$, en comparación con la fórmula de Friedewald en toda la muestra y cuando esta se estratifico por niveles de triglicéridos. Lo que es evidencia que la nueva ecuación presenta una mayor exactitud que la fórmula de Friedewald.

A pesar de que en la investigación se utilizó como método de comparación el método directo homogéneo y no la beta cuantificación que es el método de referencia, diversas investigaciones muestran que ambas técnicas presentan una buena correlación en muestras normo como dislipémicas. Todo ello hace que el método de determinación directa se constituya como una razonable alternativa al méto- do de referencia, además de presentar la ventaja de su automatización completa, lo que ocasiona una disminución apreciable de los errores en la determinación del $\operatorname{LDLC}^{(6,29)}$.

En conclusión, la nueva ecuación planteada basada en el análisis de regresión múltiple, ofrece una mayor exactitud que la fórmula de Friedewald para estimar el LDLc en diferentes niveles de triglicéridos, presenta bajo error analítico. Además de mostrar una buena concordancia con el método directo homogéneo, representa una alternativa asequible y económica cuando la cuantificación directa del LDLc no es posible.

Por otra parte, se recomienda establecer el desempeño analítico de esta ecuación en otras poblaciones, investigar su fiabilidad mediante la comparación con otras ecuaciones, así como la evaluación de su importancia clínica en patologías como diabetes, insuficiencia renal, hepatopatías, entre otras.

\section{REFERENCIAS BIBLIOGRÁFICAS}

1. Errico TL, Chen X, Martin Campos JM, Julve J, Escolà-Gil JC, Blanco-Vaca F. Mecanismo básico: estructura, función y metabolismo de las lipoproteínas plasma. Clin Invest Arterioscl. 2013;25(2):98-103.

2. GPC - Ministerio de Salud y Protección Social [Internet]. Guia de práctica clínica para la prevención, detección temprana, diagnóstico, tratamiento y seguimiento de las dislipidemias en la población mayor de 18 años. Colombia: MinSalud COLCIENCIAS; 2014 [citado el 8 de diciembre de 2015] Disponible en: http://gpc.minsalud.gov.co/ guias/Documents/Dislipidemia/GPC Dislipidemias profesionales.pdf.

3. Centro Nacional de Excelencia Tecnológica en Salud [Internet]. Diagnóstico y Tratamiento de las Dislipidemias. México: Secretaria de Salud; 2012 [Citado el 20 de enero de 2016] Disponible en: http://www.cenetec.salud.gob.mx/descargas/ gpc/CatalogoMaestro/233_GPC_Dislipidemias/ GER_Dislipidemia.pdf.

4. Nauck M, Warnick GR, Rifai N. Methods for Measurement of LDL-Cholesterol: A critical assessment of direct measurement by homogeneous assays versus calculation. Clin Chem. 2002 Feb;48(2):236-54.

5. Querales M, Cruces ME, Sánchez C, Querales M, Rojas S, Sánchez L. Medida del colesterol de lipoproteinas de baja densidad utilizando tres metodologias. Acta bioquim Clin Latinoam. 2012;46(1):31-7.

6. García GD, Martín R, Navarro M, Cabrera A, Quintana L, Aguilar JA. Evaluación de un método directo para la cuantificación de colesterol de LDL. Química Clínica. 2006;25(2):58-63.

7. Guevara CA, Criollo CP, Pineda CA. Comparison 
of low-density lipoprotein obtained from the Friedewald formula and new formulae in a heterogeneous population. Colomb Med. 2010;41:328-35.

8. Asociación Española de Biopatología Médica [Internet]. Métodos Estadisticos en la Comparación de Equipos de Laboratorio. Madrid: AEBM medicina de laboratorio; 2013 [citado el 12 de enero de 2016] Disponible en: http://www.aebm org/images/activos/documentosexternos/Mtodos Estadsticos en la Comparacin de Equipos de Laboratorio v2 2.pdf.

9. Martinez E, Gella F, Alonso N, Boned B, Canalias $F$, Izquierdo S, et al. Recomendaciones para e estudio de la veracidad en el laboratorio clínico mediante la comparación de procedimientos de medida. Documentos de la SEQC. Abril 2011.

10. Burtis CA, Ashwood ER. Tietz Fundamentals of Clinical Chemistry. 5th ed. WB Saunders Company; 2001.

11. Trinder P. Determination of glucose in blood using glucose oxidase with an alternative oxygen acceptor. Ann Clin Biochem. 1969; 6:24-27.

12. Izawa S, Okada M, Matsui $\mathrm{H}$, et. al. Medicine and Pharmaceutical Sci. 1997;37:1385-8.

13. Okada M, Matsui $H$, Ito $Y$, et al. Low density lipoprotein cholesterol can be chemically measured: A new superior method. J Lab Clin Med. 1998;132:195-201.

14. Cavada G. Concordancia Parte II: el método de Bland-Altman. Rev chil endocrinol diabetes. 2013;6(3):127-8

15. Sociedad española de medicina rural y generalista [Internet]. Abordaje común del paciente dislipémico. Madrid: SEMERGEN; 2010 [Citado el 12 de enero de 2016] Disponible en: http://projectes. camfic.cat/CAMFiC/Enviaments/Docs/CuadernoProfesorCompleto290111SEASEMERGEN.pdf.

16. Escribano A, Vega AT, Lozano JE, Álamo R, Castrodeza JJ, Lleras S. Dislipemias y riesgo cardiovascular en la población adulta de Castilla y León. Gac Sanit. 2010;24(4):282-7.

17. Nigam P. Calculated low density lipoprotein-cholesterol friedewald's formula versus other modified formulas. International Journal of Life Science and Medical Research. Apr. 2014;4(2):25-31. doi: 10.5963/LSMR0402002.

18. Gupta S, Verma M, Singh K. Does LDL-C Estimation using Anandaraja's formula give a better agreement with direct LDL-C estimation than the Friedewald's formula? Indian J Clin Biochem. 2012 Apr;27(2):127-33. doi: 10.1007/s12291011-0186-3.

19. Boshtam M, Arash M, Naderi G, Sarrafzadegan N. Is Friedewald formula a good estimation density lipoprotein level in Iranian population?. J Res Med Sci. 2012;17:519-22

20. De Cordova CM, De Cordova MM. A new accurate, simple formula for LDL-cholesterol estimation based directly measured blod lipids from a large cohort. Ann Clin biochem. 2013;50:13-9. Doi: 10.1258/acb.2012.011259.

21. Martin SS, Blaha MJ, Elshazly MB, Toth PP, Kwiterovich PO, Blumenthal RS, et al. Comparison of a novel method vs the Friedewald equation for estimating low-density lipoprotein cholesterol levels from the standard lipid profile. JAMA. 2013 November 20;310(19):2061-8. doi: 10.1001/ jama.2013.280532.

22. Saiedullah M, Chowdhury N, Khan MAH, Begum S, Rahman MR. Comparison of regression equation and Friedewald's Formula with direct measurement of low-density lipoprotein cholesterol in Bangladeshi population. J Sci Res. 2014;6(1):143-52.

23. Chowdhury N, Saiedullah M, Khan MAH, Rahman MR. Comparison of modified Friedewald's formula with direct measurement of low-density lipoprotein cholesterol in Bangladeshi population. Bangladesh Med Res Counc Bull. 2013;39:120-3.

24. Chen $Y$, Zhang $X$, Pan B, Jin X, Yao H, Chen B, et al. A modified formula for calculating low-density lipoprotein cholesterol values. Lipids Health Dis. 2010 May 21;9:52. doi: 10.1186/1476-511X-9-52.

25. Anwar M, Khan DA, Khan FA. Comparison of Friedewald formula and modified Friedewald formula with direct homogeneous assay for low density lipoprotein cholesterol estimation. J Coll Physicians surgeons Pakistan. 2014;24(1):8-12.

26. Guevara CA, Criollo CP, Pineda CA. Comparison of low-density lipoprotein obtained from the Friedewald formula and new formulae in a heterogeneous population. Colomb Med. 2010;41:328-35.

27. Expert Panel on Detection, Evaluation, and Treatment of High Blood Cholesterol in Adults. Executive Summary of The Third Report of The National Cholesterol Education Program (NCEP) Expert Panel on Detection, Evaluation, And Treatment of High Blood Cholesterol In Adults (Adult Treatment Panel III). JAMA. 2001 May 16;285(19):2486-97.

28. Saiedullah M, Chowdhury N, Khan MAH. Evaluation of the novel method and the regression equation for calculation of low-density lipoprotein cholesterol. J Enam Med Col. 2015;5(1):10-4

29. Martin SS, Blaha MJ, Elshazly MB, Brinton EA, Toth PP, McEvoy JW, et al. Friedewald-estimated versus directly measured low-desity lipoprotein cholesterol and treatment implications. J Am Coll Cardiol. 2013 Aug 20;62(8):732-9. doi: 10.1016/j. jacc.2013.01.079. 